\title{
Nuclear structure and double beta decay: Two neutrino mode
}

\author{
P. Vogel and P. Fisher \\ Physics Department, California Institute of Technology, Pasadena, California 91125
}

(Received 11 June 1985)

\begin{abstract}
The decay rate of the $2 v$ double beta decay is calculated. The effects of pairing, static quadrupole deformation, spin-isospin polarization, and the $\Delta_{33}$ isobar admixtures on the nuclear matrix elements are studied and the relation of the $2 v \beta \beta$ rate and the $\beta^{-}$and $\beta^{+}$strength is stressed. In agreement with other calculations, we predict a faster decay rate than has been observed experimentally in ${ }^{82} \mathrm{Se}$, ${ }^{130} \mathrm{Te}$, and ${ }^{150} \mathrm{Nd}$.
\end{abstract}

\section{INTRODUCTION}

Double beta decay, the process where a nucleus $(Z, A)$ undergoes a transition to $(Z+2, A)$, has long been recognized as a sensitive test of lepton number conservation, the mass of the electron neutrino, and of weak interactions involving right-handed lepton currents. There are two modes of double beta decay $(\beta \beta)$, one involving the emission of two neutrinos and another associated with zero neutrino emission. The two neutrino mode $(2 v)$ has two leptons and two antileptons, $2 \mathrm{e}^{-}+2 \bar{v}$, in the final state and is expected to occur in the standard theory in second order. The zero neutrino mode $(0 v)$ has only two electrons and no antineutrinos in the final state and requires the existence of Majorana neutrinos as well as nonvanishing neutrino mass or right-handed lepton current. In order to interpret the results of the experiments on $\beta \beta$ decay, however, one has to solve some important nuclear physics problems.

Calculation of the nuclear matrix elements in $2 v \beta \beta$ decay is the topic of this paper. The general theory of both the $2 v$ and $0 v$ processes has been reviewed many times; first by Primakoff and Rosen ${ }^{1}$ and more recently by Haxton and Stephenson. ${ }^{2}$ Very briefly, the procedure is as fol- lows: One uses the standard allowed approximation and assumes that the Gamow-Teller transitions dominate over the Fermi transitions in medium and heavy nuclei. Both the lepton and nuclear energies contribute to the energy denominators of the corresponding second order perturbation expression. For the $2 v$ mode treated here one achieves separation of the nuclear and lepton parts of the transition amplitude by replacing in the energy denominator the lepton energy of the intermediate state by its average value, i.e., by half of the available transition energy. The integration over the lepton phase space is then performed and a closed expression for the rate (or lifetime) is obtained.

There is a general consensus ${ }^{2-4}$ that these approximations do not result in an appreciable loss of accuracy. There is also an agreement that the nuclear matrix elements responsible for the $0^{+} \rightarrow 0^{+} 2 v$ and $0 v$ decays are related. Thus, a good understanding of the $2 v$ lifetimes is a necessary condition for the correct interpretation of the fundamentally more important $0 v$ process. In the following we shall consider only the $2 v$ process and leave the calculation of the $0 v$ decay rate for future papers.

The half-life of the $2 v$ decay between two $0^{+}$ground states of even-even nuclei is given, in the approximations listed above, by the formula

$$
\left[T_{1 / 2}^{2 v}\left(0^{+} \rightarrow 0^{+}\right)\right]^{-1}=G_{\mathrm{GT}}\left(E_{\mathrm{max}}, Z\right)\left|\sum_{m} \frac{\left\langle f\left|\sum_{l} \sigma_{l} \tau_{l}^{+}\right| m\right\rangle\left\langle m\left|\sum_{k} \sigma_{k} \tau_{k}^{+}\right| i\right\rangle}{E_{m}-\left(M_{i}+M_{f}\right) / 2}\right|^{2} .
$$

Here $|i\rangle(|f\rangle)$ is the initial (final) nucleus in the $0^{+}$ ground state with the mass $M_{i}\left(M_{f}\right) ;|m\rangle$ are the $1^{+}$ states of the intermediate odd-odd nucleus with energies $E_{m}, \sigma_{l}$ is the usual Pauli spin operator for the nucleon $\bar{l}$, and $\tau_{l}^{+}$is the isospin raising operator changing a neutron into a proton. (The energy denominators are in units of $m_{e} c^{2}$.) The function $G_{\mathrm{GT}}\left(E_{\max }, Z\right)$ results from lepton phase space integration; it contains all of the relevant constants including the coupling strength $\left(G g_{A}\right)^{4}$ as well as the relativistic Coulomb terms. This function has been calculated for several cases by Doi et al. ${ }^{3}$ A complete set of $G_{\mathrm{GT}}$ values can be deduced from Table 1A of Ref. 2 using the $\tau_{1 / 2}^{2 v}$ values given there and the relation

$$
G_{\mathrm{GT}}=\left(1.2 A / \tau_{1 / 2}^{2 v}\right) \mathrm{yr}^{-1}
$$

[The factor 1.2 $\mathrm{A}$ arises from the estimate of the average energy denominator used in closure approximation, $1.2 A=\left(1.12 A^{1 / 2} \mathrm{MeV} / 2 m_{e} c^{2}\right)^{2}$.] Note that the small systematic differences in $G_{\mathrm{GT}}(2-6 \%)$ between Refs. 2 and 3 are of no concern at the present time.

In the following we shall evaluate the sum in Eq. (1) in a series of succesive approximations. We are not going to use the customary closure approximation which removes 
the summation over the intermediate states in (1). Instead, the summation will be performed explicitly. We shall study the dependence and sensitivity of the results on the various empirical parameters. Whenever possible, we shall relate our results to the other experimentally accessible information, primarily the $\beta^{-}$and $\beta^{+}$strength functions.

Such a comparison is possible because the matrix element $\left\langle m\left|\sum_{l} \sigma_{l} \tau_{l}^{+}\right| i\right\rangle$ in Eq. (1) describes the amplitude of the Gamow-Teller $\beta^{-}$transition connecting the initial state with the corresponding $1^{+}$state of the intermediate odd-odd nucleus. Alternatively, the same matrix element describes the amplitude of the ( $p, n)$ forward angle reaction on the initial nucleus at a suitable energy. ${ }^{5}$ Similarly, the matrix element $\left\langle f\left|\sum_{l} \sigma_{l} \tau_{l}^{+}\right| m\right\rangle$ is proportional to the $\beta^{+}$transition amplitude connecting the final state with the same intermediate $1^{+}$state, or the amplitude of the $(n, p)$ reaction on the final nucleus. Knowledge of the experimental $f t$ values or of the $(\mathrm{p}, \mathrm{n})$ and $(\mathrm{n}, \mathrm{p})$ cross sections makes it possible, therefore, to obtain the magnitude, although not the sign, of the corresponding terms in Eq. (1).

\section{REVIEW OF EXPERIMENTAL DATA ON $2 v \beta \beta$ DECAY}

There are two sources of experimental information on $2 v \beta \beta$ decay, the direct counting technique and the geochemical technique. The laboratory counter experiments allow one to separate the $2 v$ and $0 v$ modes. Positive evidence, with some caveats, exists only in the case of ${ }^{82} \mathrm{Se}$. Restrictive limits have been obtained in several other cases. The geochemical measurements determine the total abundance of the final nucleus, giving, therefore, the total decay rate, and a lower limit on the $2 v$ mode partial lifetime.

The existing experimental data are collected in Table I. From the lifetimes there and the known $G_{G T}$ values, we extract the relevant nuclear structure part,

$$
M_{\mathrm{GT}}=\sum_{m} \frac{\left\langle f\left|\sum_{l} \sigma_{l} \tau_{l}^{+}\right| m\right\rangle\left\langle m\left|\sum_{k} \sigma_{k} \tau_{k}^{+}\right| i\right\rangle}{E_{m}-\left(M_{i}+M_{f}\right) / 2} .
$$

Calculation of this quantity is the goal of the present paper. For comparison we also show in Table I the values calculated in Ref. 2 . These values are $\approx 3$ times larger than the experimental ones for ${ }^{82} \mathrm{Se}$ and $\approx 12$ times larger for ${ }^{130} \mathrm{Te}$. The decay of ${ }^{82} \mathrm{Se}$ has been studied by both techniques; the counter limit and the geochemical rates are within a factor of 2 of each other. It should be noted that the systematic effects favor longer lifetimes in counter studies (background) and shorter lifetimes in geochemical studies (gas retention), thus conveniently bracketing the ${ }^{82} \mathrm{Se}$ rate. The ${ }^{130} \mathrm{Te}$ decay rate has been determined only in geochemical measurements.

\section{PAIRING CORRELATIONS}

Pairing correlations play a double role in double beta decay. The extra stability of the even-even nuclei caused by the pairing correlations makes possible the rather frequent appearance of the $\beta \beta$ decay candidates.

In most cases, ${ }^{48} \mathrm{Ca}$ and ${ }^{100} \mathrm{Mo}$ being notable exceptions, the neutron Fermi level of the initial nucleus and the proton Fermi level of the final nucleus are in different shells and the $\beta \beta$ decay rate therefore vanishes in the extreme nuclear shell model. However, the smearing of the occupation probabilities caused by the pairing correlations allows the $\beta \beta$ decay to proceed. Moreover, the different subshells contribute coherently to the total matrix element. ${ }^{11}$ Deformation effects also remove some of the selection rules of the single-particle shell model, ${ }^{11}$ though, as we shall see below, the $\beta \beta$ decay rate usually depends only weakly on the deformation.

To see the effect of pairing one replaces in Eq. (1) the energy denominators by an average value (closure approximation) to obtain

$$
M_{\mathrm{GT}}=\frac{1}{\overline{\Delta E}}\left\langle f\left\|\sum_{k, l} \sigma_{k} \cdot \sigma_{l} \tau_{k}^{+} \tau_{l}^{+}\right\| i\right\rangle \equiv \frac{\mathbf{M}_{\mathrm{GT}}}{\overline{\Delta E}} .
$$

In the standard Bardeen-Cooper-Schrieffer (BCS) approximation one gets

$$
\mathbf{M}_{\mathrm{GT}}=-\sum_{j_{\mathrm{n}} j_{\mathrm{p}}}\left\langle j_{\mathrm{p}}\|\sigma\| j_{\mathrm{n}}\right\rangle^{2} v_{j_{\mathrm{n}}}^{(i)} u_{j_{\mathrm{p}}}^{(i)} v_{j_{\mathrm{p}}}^{(f)} u_{j_{n}}^{(f)}
$$

where $j_{\mathrm{p}}\left(j_{\mathrm{n}}\right)$ also includes the other relevant quantum numbers. Noting that, typically, in Eq. (4) the operator $\sigma$ connects states of which one, but not both, are near the Fermi level, we conclude that $\mathbf{M}_{\mathrm{GT}}$ is proportional to the pairing gap $\Delta$. This conclusion is borne out by the nu-

\begin{tabular}{|c|c|c|c|c|c|c|}
\hline Parent nucleus & ${ }^{48} \mathrm{Ca}$ & ${ }^{76} \mathrm{Ge}$ & ${ }^{82} \mathrm{Se}$ & ${ }^{82} \mathrm{Se}$ & ${ }^{130} \mathrm{Te}$ & ${ }^{150} \mathrm{Nd}$ \\
\hline$\overline{T_{1 / 2 \operatorname{expt}}^{(2 y)}(\mathrm{yr})}$ & $>3.6 \times 10^{19}$ & $>2.8 \times 10^{19}$ & $>4 \times 10^{19}$ & $(1.45 \pm 0.15) \times 10^{20}$ & $(2.6 \pm 0.3) \times 10^{21}$ & $>1.3 \times 10^{19}$ \\
\hline Reference & 6 & 7 & 8 & 9 & 9 & 10 \\
\hline$G_{\mathrm{GT}}\left(\mathrm{yr}^{-1}\right)$ & $4.0 \times 10^{-17}$ & $1.3 \times 10^{-19}$ & $4.5 \times 10^{-18}$ & $4.5 \times 10^{-18}$ & $4.8 \times 10^{-18}$ & $1.2 \times 10^{-16}$ \\
\hline$\left(M_{\mathrm{GT}}\right)^{\text {expt }}$ & $<2.6 \times 10^{-2}$ & $<0.52$ & $<7.5 \times 10^{-2}$ & $3.9 \times 10^{-2}$ & $8.9 \times 10^{-3}$ & $<2.5 \times 10^{-2}$ \\
\hline$\left(M_{\mathrm{GT}}\right)^{\mathrm{calc}}$, Ref. 2 & $2.9 \times 10^{-2}$ & 0.14 & $9.2 \times 10^{-2}$ & $9.2 \times 10^{-2}$ & 0.11 & \\
\hline$\left(M_{\mathrm{GT}}\right)^{\text {calc }}$, present work $^{\mathrm{a}}$ & & $6.6 \times 10^{-2}$ & & $8.2 \times 10^{-2}$ & 0.09 & $6.4 \times 10^{-2}$ \\
\hline
\end{tabular}

TABLE I. Experimental data on the $2 v \beta \beta$ decay. (The calculated $M_{\mathrm{GT}}$ values of Ref. 2 and of the present work are shown for comparison in the last two lines.)

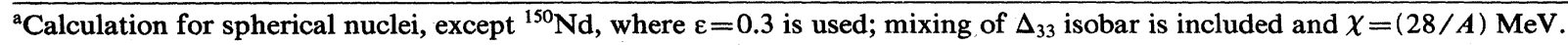




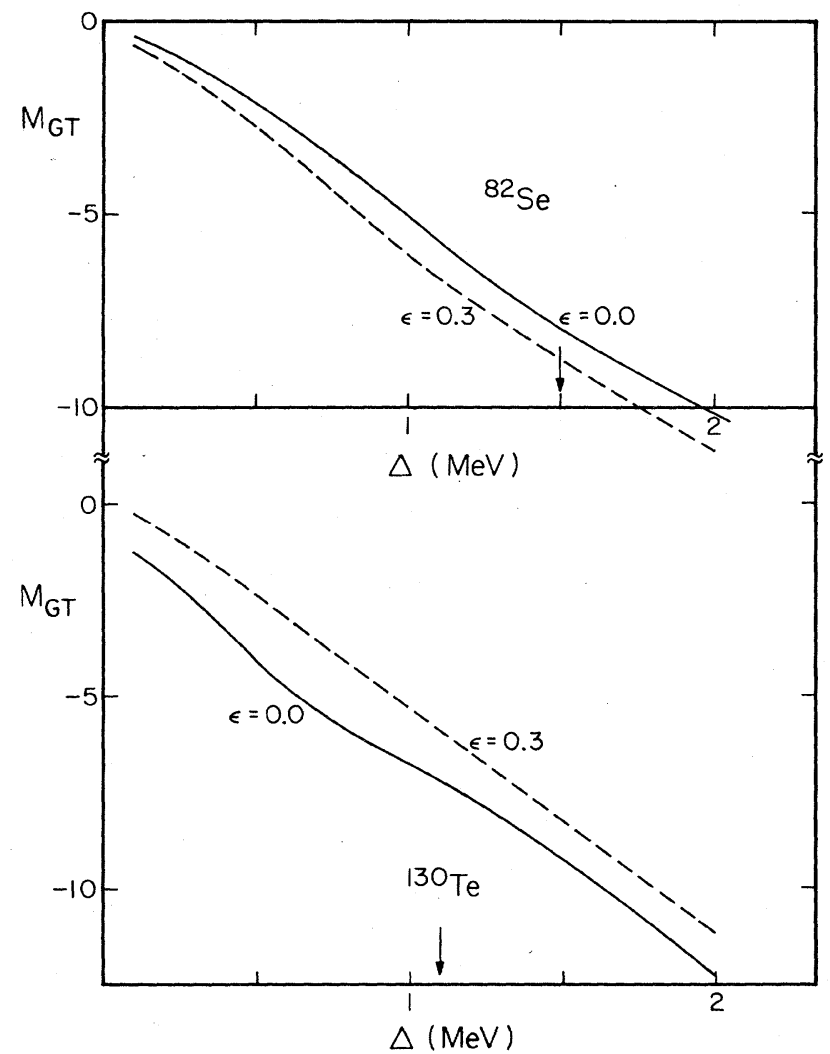

FIG. 1. The matrix element $\mathbf{M}_{\mathrm{GT}}$ as a function of the pairing gap $\Delta$ for ${ }^{130} \mathrm{Te}$ (lower part) and ${ }^{82} \mathrm{Se}$ (upper part) and for two values of the deformation parameter $\varepsilon$. Two oscillator shells were used in the calculation. The arrows indicate experimental $\Delta$ values from pairing energies. (To compare with Refs. 2 and 12 , one should change the sign and divide by 2 .)

merical results shown in Fig. 1. They agree well with the results of Ref. 12 (except the apparent mistake there for $\eta=0$ ). Note that the terms in Eq. (4) contribute coherently and the matrix elements far exceed the naive expectation $\left|\sigma_{k} \cdot \sigma_{l}\right| \leq 3$.

It is also worth noting that states relatively far away from the Fermi level give an important contribution in Eq. (4). Therefore, when a limited configuration space is used (less than two oscillator shells) a severe underestimate of $\mathbf{M}_{\mathrm{GT}}$ results.

The $u$ and $v$ factors in Eq. (4) refer to the initial and final nucleus, respectively. For consistency one should multiply the result by the corresponding overlap factor ${ }^{11}$ which is, however, usually close to unity. Huffman ${ }^{13}$ discusses the problem of the number and isospin nonconservation in the BCS treatment and shows that the beta decay operator does not contain spurious parts up to second order in the parameter $\Omega^{-1 / 2}\left[\Omega=\left(j+\frac{1}{2}\right)\right.$ is the number of pairs].

\section{SPIN-ISOSPIN POLARIZATION FORCE}

It has long been known that pairing alone cannot explain the $\beta^{-}$and $\beta^{+}$rates; the calculated $f t$ values are consistently smaller than the experimental ones. Halbleib and Sorensen, ${ }^{14}$ following the earlier suggestion of Ikeda,
Fuji, and Fujita, ${ }^{15}$ showed that a better agreement is obtained with a schematic neutron-proton "Gamow-Teller" force treated within the random phase approximation (RPA) formalism. The same force successfully explains the main features of the giant GT resonance (see Ref. 5 and references therein).

Huffmann ${ }^{13}$ applied the approach of Ref. 14 to the calculation of $\beta \beta$ decay and showed that a considerable reduction of the rate results. More recently, Klapdor and collaborators ${ }^{16}$ performed similar calculations with somewhat modified treatment. The formalism of Ref. 14 has been extended to the case of deformed nuclei by Krumlinde and Moller. ${ }^{17}$

Here we review only the main features of the formalism. ${ }^{14}$ The model space in our case contains two complete oscillator shells. In the numerical calculation we use the eigenvalues and eigenfunctions of the modified harmonic oscillator; ${ }^{17}$ the results do not change appreciably when Woods-Saxon potential eigenvalues are used instead. The pairing gap $\Delta$ is taken from the experimental pairing energies and the chemical potentials, $\lambda_{p}$ and $\lambda_{n}$, are then determined from the usual condition of the average number conservation.

First, we introduce quasiparticles (operators $\alpha, \alpha^{\dagger}$ ) by the canonical transformation and then define the neutron-proton pair operators

$$
A_{J M}^{\dagger}\left(j_{\mathrm{p}}, j_{\mathrm{n}}\right)=\sum_{m_{\mathrm{p}}, m_{\mathrm{n}}}\left\langle j_{\mathrm{p}} m_{\mathrm{p}} j_{\mathrm{n}} m_{\mathrm{n}} \mid J M\right\rangle \alpha_{j_{\mathrm{p}} m_{\mathrm{p}}}^{\dagger} \alpha_{j_{\mathrm{n}} m_{\mathrm{n}}}^{\dagger} .
$$

The RPA signifies in this case that the boson commutation relations should be used for $A, A^{\dagger}$ and that all terms which cannot be expressed in terms of these operators should be neglected. Thus, for example, the $\beta^{-}$transition operator is written as

$$
\begin{aligned}
\beta_{\mu}^{-}= & \sum_{\mathrm{p}, \mathrm{n}}\left\langle\mathrm{p}\left|\sigma_{\mu}\right| \mathrm{n}\right\rangle a_{\mathrm{p}}^{\dagger} a_{\mathrm{n}} \\
=\frac{1}{\sqrt{3}} \sum_{j_{\mathrm{p}} j_{\mathrm{n}}}\left\langle j_{\mathrm{p}}\|\sigma\| j_{n}\right\rangle\left[v_{j_{\mathrm{n}}} u_{j_{\mathrm{p}}} A_{1 \mu}^{\dagger}\left(j_{\mathrm{p}} j_{\mathrm{n}}\right)\right. & \left.\quad-(-1)^{\mu} u_{j_{\mathrm{n}}} v_{j_{\mathrm{p}}} A_{1-\mu}\left(j_{\mathrm{p}} j_{\mathrm{n}}\right)\right] \\
= & \sum_{k}\left[\sigma_{k} A_{1 \mu}^{\dagger}(k)-(-1)^{\mu} \bar{\sigma}_{k} A_{1-\mu}(k)\right],
\end{aligned}
$$

using the label $k$ for the pair of indices $j_{\mathrm{p}} j_{\mathrm{n}}$ and obvious notation for $\sigma_{k}$ and $\bar{\sigma}_{k}$. (The $\beta^{+}$operator is obtained by the substitution $\sigma_{k} \rightarrow \bar{\sigma}_{k}$.)

Next, we introduce the "Gamow-Teller" spin-isospin polarization force

$$
V_{\mathrm{GT}}=2 \chi \beta^{-} \cdot \beta^{+},
$$

where $\mathcal{X}$ is the empirical interaction strength parameter to be determined later. The $1^{+}$states in the odd-odd nucleus are described by the phonon operators

$$
\Gamma_{\mu}^{+}(l)=\sum_{k}\left[x_{k}^{(l)} A_{1 \mu}^{\dagger}(k)-(-1)^{\mu} y_{k}^{(l)} A_{1-\mu}(k)\right],
$$

where the amplitudes $x$ and $y$, as well as the energies $\omega^{(l)}$, are determined by the solution of the equation of motion for the operators $\Gamma_{\mu}(l)$. The ground state of the even-even 
nucleus is the vacuum of all $\Gamma_{\mu}(l)$ operators, $\Gamma_{\mu}(l) \mid$ g.s. $\rangle=0$.

The $\beta^{-}$matrix element for the transition into the $n$th $1^{+}$state with energy $\omega^{(l)}$ is equal to

$$
\beta_{(\bar{l})}=\sum_{k}\left(x_{k}^{(l)} \sigma_{k}-y_{k}^{(l)} \bar{\sigma}_{k}\right) \text {. }
$$

Again the corresponding $\beta^{+}$matrix element is obtained by replacing $\sigma_{k} \rightarrow \bar{\sigma}_{k}$.

The sum rule

$$
\sum_{l}\left[\left(\beta_{(l)}^{-}\right)^{2}-\left(\beta_{(l)}^{+}\right)^{2}\right]=(N-Z)
$$

is independent of the coupling strength $\chi$. [Note that the usual $3(N-Z)$ rule is obtained by summing over the $3 \mathrm{~m}$ projections of the $1^{+}$state.]

The value of $\chi$ is determined from the requirement that the calculation reproduces the known energies of the giant GT resonance. The fit is performed in a following way: Our Hamiltonian does not contain the Coulomb and symmetry energies and thus cannot reproduce the ground state masses. To compensate for this shortcoming we add to all energies $\omega^{(l)}$ the difference between the experimental nuclear mass splitting and the calculated mass splitting (i.e., the minimal two-quasiparticle energy). Only then can we perform the fit to obtain

$$
\chi=\frac{23}{A} \mathrm{MeV} \text {. }
$$

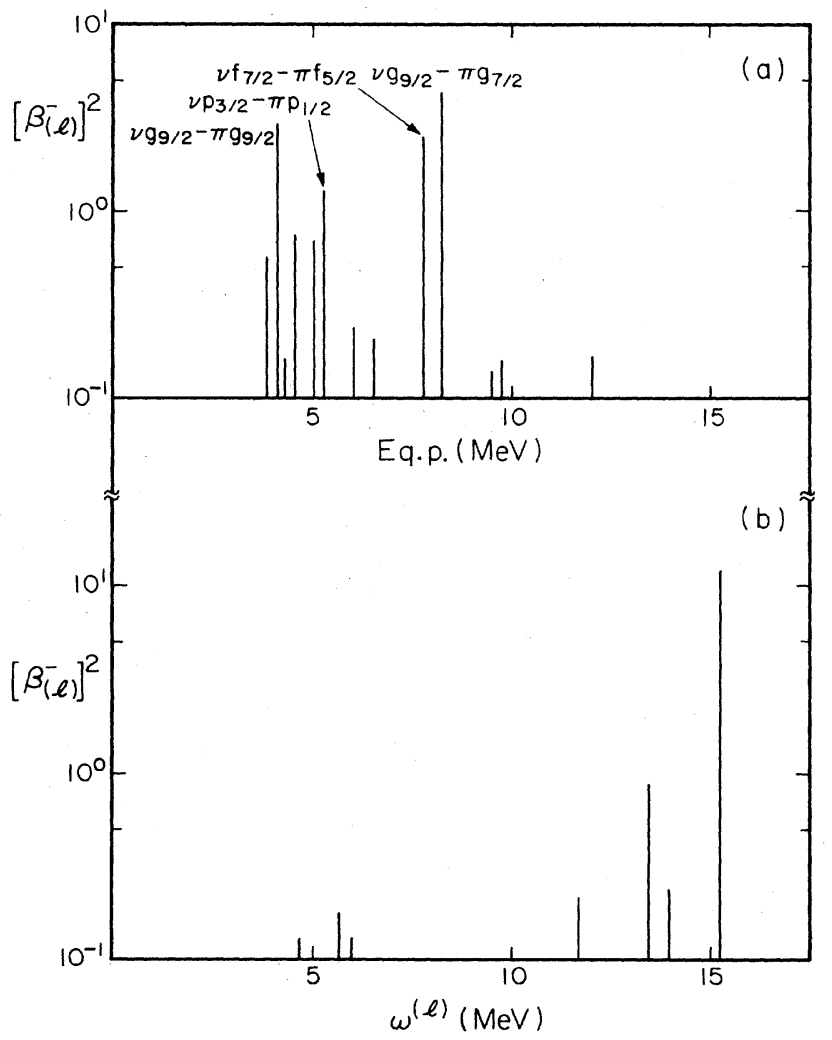

FIG. 2. (a) $\beta^{-}$strength for ${ }^{82} \mathrm{Se}$ without interaction; (b) with interaction and $\chi=(23 / A) \mathrm{MeV}$, Pairing gap $\Delta=1.5 \mathrm{MeV}$ was used.

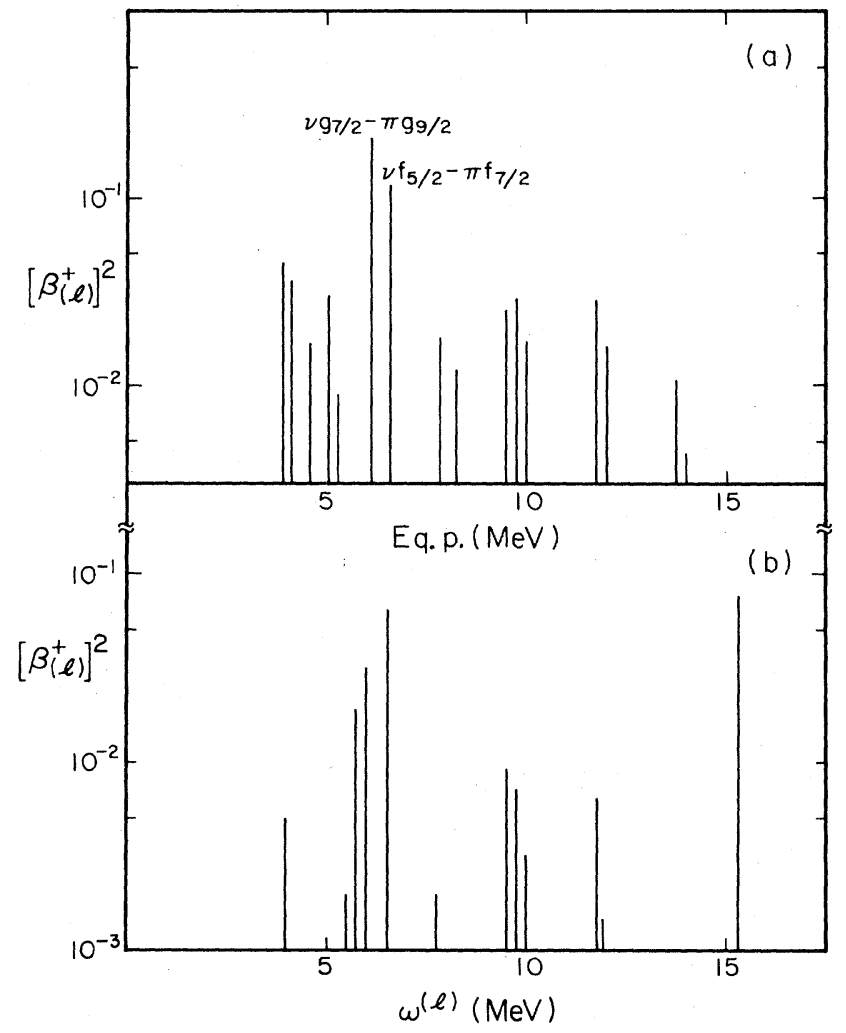

FIG. 3. (a) $\beta^{+}$strength for ${ }^{82} \mathrm{Se}$ without interaction; (b) with interaction and $\chi=(23 / A) \mathrm{MeV}$.

This procedure reproduces the giant GT resonance energies within $1 \mathrm{MeV}$ for a number of nuclei between ${ }^{48} \mathrm{Ca}$ and ${ }^{208} \mathrm{~Pb}$. Equation (11) agrees with similar adjustments made in Ref. 5 . Note, however, that variations by $\approx 10 \%$ cannot be excluded.

The $\beta^{-}$and $\beta^{+}$strengths for ${ }^{82} \mathrm{Se}$ are shown in Figs. 2 and 3 for the case of noninteracting quasiparticles and for the case of an interaction with $\chi=(23 / A) \mathrm{MeV}$. Note that the $\beta^{-}$strength is essentially conserved, but is shifted up in energy and concentrated in the GT giant resonance. On the other hand, the $\beta^{+}$strength, which is much smaller to begin with, is further reduced by a factor of $\approx 3$ and remains spread among several states. It turns out that the giant GT state often contributes a relatively large fraction of the total $\beta^{+}$strength.

The rate of the $2 v \beta \beta$ decay is determined by the quantity $M_{\mathrm{GT}}$ in Eq. (2), or in our notation:

$$
M_{\mathrm{GT}}=\sum_{l} \frac{\beta_{(l)}^{-} \beta_{(l)}^{+}}{\omega^{(l)}+\Delta E},
$$

where

$$
\Delta E=M_{\text {odd-odd }}^{\text {atomic }}-M_{\text {initial }}^{\text {atomic }}+\frac{T_{\max }}{2}-\left(E_{\mathrm{qp}}^{\text {neut }}+E_{\mathrm{qp}}^{\text {prot }}\right)_{\min } .
$$

Here the index "atomic" denotes the atomic mass, $T_{\max }$ is the kinetic energy available to the outgoing leptons, and the sum extends over all solutions of the RPA equation of motion. 


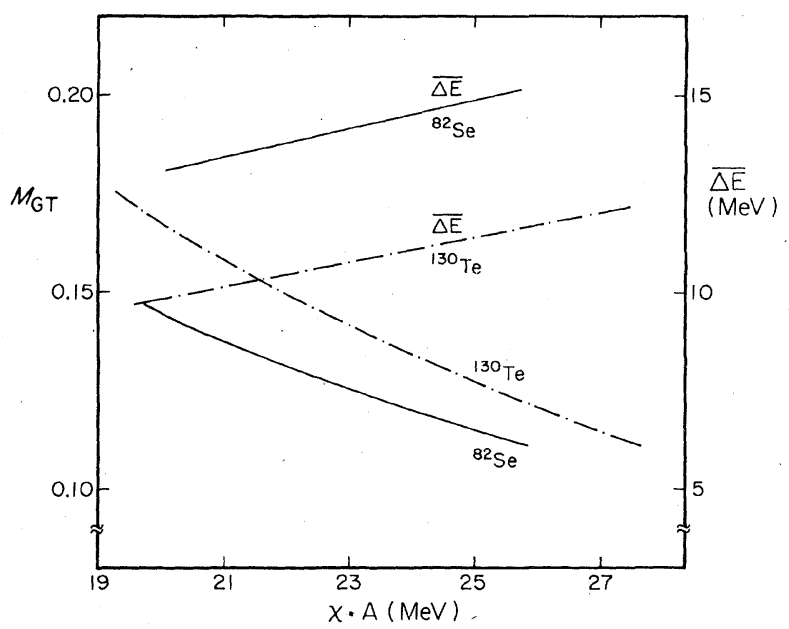

FIG. 4. The quantities $M_{\mathrm{GT}}$ [Eq. (12), left scale] and the average energy denominators $\overline{\Delta E}$ [Eq. (3), right scale] are shown as a function of the strength $\chi$ for ${ }^{82} \mathrm{Se}$ (full curves, $\Delta=1.5$ $\mathrm{MeV}$ ) and ${ }^{130} \mathrm{Te}$ (dot-and-dashed curves, $\Delta=1.0 \mathrm{MeV}$ ).

In Eq. (12) one should use the quantities $\beta_{(l)}$ corresponding to the initial nucleus and $\beta_{(l)}^{+}$corresponding to the final nucleus. However, in the calculation both amplitudes necessarily involve the same even-even core. By replacing the initial nucleus by the final one the quantity $M_{\mathrm{GT}}$ increases by about $40 \%$. In order to preserve the essential simplicity of our approach we chose an intermediate solution in which the RPA equations are solved only once but the quantities $\sigma_{k}$ [Eqs. (6) and (9)] refer to the initial nucleus, while $\bar{\sigma}_{k}$ refer to the final one. The results for ${ }^{82} \mathrm{Se}$ and ${ }^{130} \mathrm{Te}$ are shown in Fig. 4.

Compared with the free quasiparticle result, the quantity $M_{\mathrm{GT}}$ is reduced by a factor of about 5 . Both the overall reduction of the $\beta^{+}$strength and the upward energy shift of the $\beta^{-}$strength contribute to this reduction. The giant GT state typically gives the largest contribution to the sum (12). The average energy denominators $\overline{\Delta E}$ are also shown in Fig. 4; they agree only in a crude way with the smooth estimate $1.12 A^{1 / 2} \mathrm{MeV}^{2}$. Note that the quantity $\overline{\Delta E}$ defined by $\mathrm{Eq}$. (3) does not enter directly into the calculation of the $\beta \beta$ decay rate.

There are two parameters involved, the pairing gap $\Delta$ and the interaction strength $\chi$. Both are fixed by experimental quantities unrelated to $\beta \beta$ decay. It is encouraging that our results are close to those of Haxton and Stephenson, ${ }^{2}$ which are based on a formally quite different approach. Our results also agree with some of the calculations of Ref. 16 (when similar input parameters are used) in which the problems related to the BCS nucleon number nonconservation are avoided by projection.

Very little is known experimentally about the $\beta^{+}$ strength function in nuclei with large neutron excess, such as the $\beta \beta$ decay candidates. Our calculations show that the decay rate depends sensitively on the details of the $\beta^{+}$ strength distribution. Any information about it, for example study of the $(n, p)$ or possibly $\left(\gamma, \pi^{+}\right)$reactions, would be a welcome addition.

\section{DEFORMATION EFFECTS}

Quadrupole deformation changes the selection rules of the single-particle and interaction matrix elements. It also removes the spherical subshell degeneracy, sharply increasing the number of intermediate $1^{+}$states. Zamick and Auerbach ${ }^{11}$ as well as Haxton and Stephenson ${ }^{12}$ discussed the effect of deformation on the matrix elements for noninteracting quasiparticles calculated in the closure approximation. Here we include the deformation effect in the full RPA treatment.

The generalization of the RPA equations to the deformed case is straightforward, and the corresponding formulas can be found in Ref. 17. For the case of axial symmetry considered here, the angular momentum projection on the symmetry axis, $K$, remains a good quantum number and so one can solve separately the RPA equations of motion for $K=0$ and $|K|=1$. The $K=0$ states exhaust $\frac{1}{3}$ of the sum rule (10) and the $|K|=1$ states contain the remaining $\frac{2}{3}$.

The numerical calculations were performed with the modified harmonic oscillator wave functions and eigenvalues. ${ }^{17}$ The chemical potentials of the initial nucleus were used throughout. As explained above, this causes a slight reduction of the resulting matrix element.

A representative example of the deformation dependence is shown in Fig. 5. Generally, we find that the closure matrix element is essentially independent of deformation while the sum including the energy denominators depends weakly on deformation, usually with a minimum at $\varepsilon=0$. Thus the increased number of intermediate states does not lead to cancellations between the terms of the sum (12). The only permanently deformed nucleus we studied, ${ }^{150} \mathrm{Nd}$, is an exception; in it the quantity $M_{\mathrm{GT}}$ is maximal for $\varepsilon=0$ and decreases for $|\varepsilon| \neq 0$.

In a related development, Klapdor and $\mathrm{Grotz}^{16}$ considered the effect of zero point motion associated with the

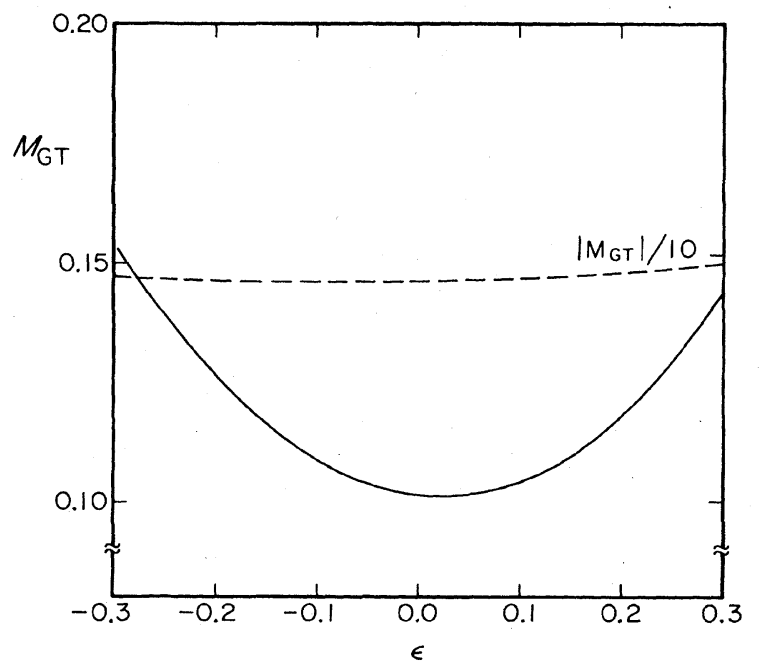

FIG. 5. Deformation dependence of the $\beta \beta$ decay matrix element for ${ }^{82} \mathrm{Se}$. Calculation performed with $\Delta_{\mathrm{p}}=\Delta_{\mathrm{n}}=1.5 \mathrm{MeV}$ and $\chi=(23 / A) \mathrm{MeV}$. The closure matrix element $\mathrm{M}_{\mathrm{GT}}$ (divided by 10 ) is shown as a dashed line. 
quadrupole degrees of freedom. They found a large reduction of the double beta decay rate for the case of ${ }^{130} \mathrm{Te}$. Direct comparison with our result is difficult, however, because Ref. 16 used smaller (perhaps unrealistically small) values of the pairing gap $\Delta$.

\section{INCLUSION OF THE $\Delta_{33}$ ISOBAR}

Only approximately half of the sum rule (10) is observed in the $(p, n)$ reaction. ${ }^{5}$ It has been suggested ${ }^{18}$ that the mixing of the $1^{+}$states of the odd-odd nucleus with the $\Delta_{33}$ isobar-nucleon hole states is responsible for this "missing strength" effect.

We shall consider the influence of the $\Delta_{33}$ nucleon-hole states on $\beta \beta$ decay using the simplest quark model of the $\Delta_{33}$ and the nucleon. The problem has been addressed before. ${ }^{11,19}$ However, the basic Eqs. (5) of Ref. 19 have incorrect pairing factors and it is not clear whether these errors were corrected in the numerical calculations.

Two $\Delta_{33}$ nucleon-hole states at approximately $300 \mathrm{MeV}$ in the intermediate odd-odd nucleus have to be considered:

$$
|1\rangle=\frac{1}{\sqrt{2}}\left(\left|\Delta^{++} \mathrm{p}^{-1}\right\rangle_{i}+\left|\Delta^{+} \mathrm{n}^{-1}\right\rangle_{i}\right)
$$

and

$$
|2\rangle=\frac{1}{\sqrt{2}}\left(\left|\Delta^{0} \mathrm{p}^{-1}\right\rangle_{f}+\left|\Delta^{-} \mathrm{n}^{-1}\right\rangle_{f}\right) .
$$

Here the indices $i$ and $f$ denote $\Delta_{33}$ nucleon-hole states in the initial and final nucleus. The energy differences of the different single-nucleon states are small compared to 300 $\mathrm{MeV}$ and therefore were neglected in constructing the states (13) and (14).

The $\beta^{-}$and $\beta^{+}$operators have the following matrix elements:

$$
\begin{aligned}
& \left\langle 1\left|\beta^{-}\right| i\right\rangle=\frac{2 \sqrt{2}}{5}(N+3 Z)^{1 / 2}, \\
& \left\langle 2\left|\beta^{+}\right| f\right\rangle=\frac{2 \sqrt{2}}{5}(Z+3 N)^{1 / 2},
\end{aligned}
$$

and

$$
\left\langle 1\left|\beta^{+}\right| f\right\rangle=\left\langle 2\left|\beta^{-}\right| i\right\rangle=0 .
$$

The states $|1\rangle$ and $|2\rangle$ are treated now as any other $1^{+}$ states of the intermediate nucleus in the RPA equations of motion. That is, we assume that the same interaction of Eq. (7) affects them.

Due to the mixing of low-lying states with state $|1\rangle$, a part $(\approx 35 \%)$ of the $\beta^{-}$strength is shifted to $300 \mathrm{MeV}$. In order to keep the giant GT state at its experimentally determined energy, we renormalize the coupling constant $\chi$ as in Refs. 5 and 18 and obtain

$$
\chi_{\mathrm{eff}}=\frac{28}{A} \mathrm{MeV}
$$

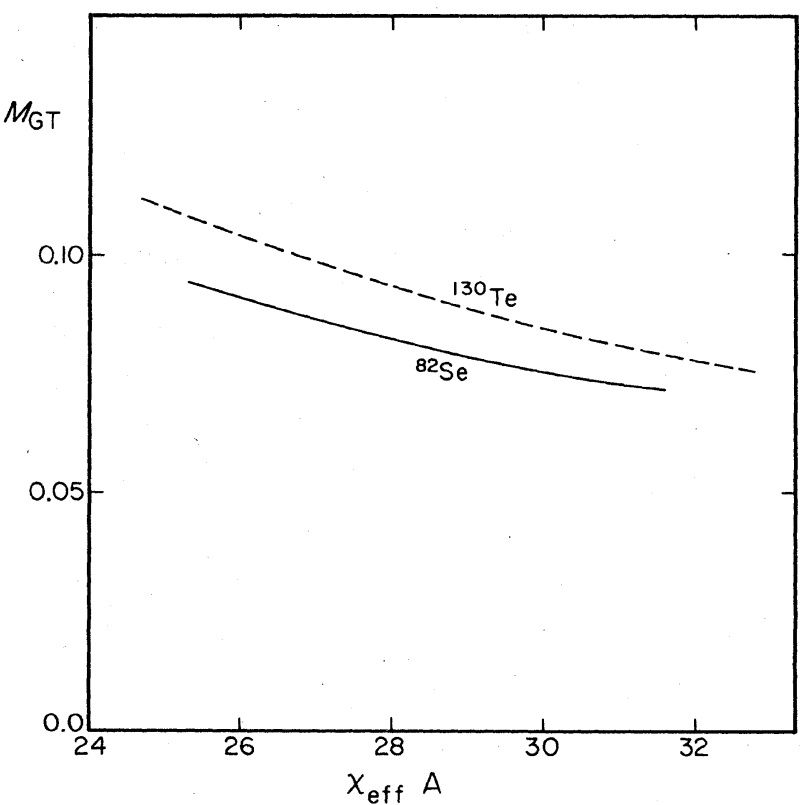

FIG. 6. The quantities $M_{\mathrm{GT}}$, Eq. (12), are shown as a function of the renormalized strength $\chi_{\text {eff }}$, for ${ }^{82} \mathrm{Se}$ (full curves, $\Delta=1.5 \mathrm{MeV}$ ) and ${ }^{130} \mathrm{Te}$ (dashed curves, $\Delta=1.0 \mathrm{MeV}$ ). The effect of the $\Delta_{33}$ isobar was included as described in the text.

In a similar way the low-lying $\beta^{+}$strength is reduced due to the mixing with state $|2\rangle$.

The $\beta \beta$ matrix element, with or without the closure approximation, is obtained by adding the contribution of all $1^{+}$states including the two mixed states at $\approx 300 \mathrm{MeV}$. These latter states, however, do not directly contribute to the $\beta \beta$ decay as a consequence of relation (16). This is so despite the fact that after the $V_{\mathrm{GT}}$ interaction has been included these high-lying states carry large $\beta^{-}$and $\beta^{+}$ strengths simultaneously.

The resulting $M_{\mathrm{GT}}$ values are shown in Fig. 6. The reduction is $\approx 30 \%$, insufficient to explain the experimental data, but large enough to be considered in quantitative calculations. It should be noted that alternative explanations of the missing strength have been proposed. ${ }^{20}$ In them the missing strength is shifted to $10-45 \mathrm{MeV}$ by mixing the two-particle-two-hole excitations. It is difficult to estimate the effect of such mixing on the $\beta \beta$ decay rate.

\section{OTHER NUCLEI AND CONCLUSIONS}

Table II shows the calculated matrix elements and other related quantities for several $2 v \beta \beta$ decay candidates. A few comments should be made: As mentioned above, ${ }^{150} \mathrm{Nd}$ is a good rotor and the matrix elements are maximal for the spherical case, decreasing monotonically with increasing deformation. The reduction is by about a factor of 2 for the realistic value $\varepsilon=0.3$. Nevertheless, the calculated lifetime is considerably smaller than the experimental limit, ${ }^{10}$ similar to the trend noted in ${ }^{82} \mathrm{Se}$ and ${ }^{130} \mathrm{Te}$. 
TABLE II. Results of the present calculation: matrix elements, lifetimes, and related quantities.

\begin{tabular}{|c|c|c|c|c|c|c|c|c|}
\hline Nucleus $^{a}$ & ${ }^{76} \mathrm{Ge}$ & ${ }^{82} \mathrm{Se}$ & ${ }^{100} \mathrm{Mo}$ & ${ }^{128} \mathrm{Te}$ & ${ }^{130} \mathrm{Te}$ & ${ }^{136} \mathrm{Xe}$ & ${ }^{150} \mathrm{Nd}(\varepsilon=0.0)$ & ${ }^{150} \mathrm{Nd}(\varepsilon=0.3)$ \\
\hline$T_{0}(\mathrm{MeV})$ & 2.05 & 3.00 & 3.03 & 0.87 & 2.53 & 2.48 & 3.37 & 3.37 \\
\hline \multirow[t]{2}{*}{ Pairing $\Delta(\mathrm{MeV})$} & 1.4 & 1.5 & $1.5(\mathrm{p})$ & 1.0 & 1.0 & 1.0 & 1.0 & 1.0 \\
\hline & & & $1.2(\mathrm{n})$ & & & & & \\
\hline$G_{\mathrm{GT}}\left(\mathrm{yr}^{-1}\right)$ & $1.3 \times 10^{-19}$ & $4.5 \times 10^{-18}$ & $9.0 \times 10^{-18}$ & $8.5 \times 10^{-22}$ & $4.8 \times 10^{-18}$ & $4.6 \times 10^{-18}$ & $1.1 \times 10^{-16}$ & $1.1 \times 10^{-16}$ \\
\hline$M_{\mathrm{GT}}[\chi=(23 / A) \mathrm{MeV}]$ & 0.10 & 0.125 & 0.35 & 0.16 & 0.14 & 0.12 & 0.20 & 0.10 \\
\hline$M_{\mathrm{GT}}{ }^{\mathrm{b}}[\chi=(28 / A) \mathrm{MeV}]$ & 0.066 & 0.082 & 0.23 & 0.10 & 0.09 & 0.098 & 0.13 & 0.064 \\
\hline$\overline{\Delta E}(\mathrm{MeV})$ & 14.7 & 14.1 & 6.1 & 10.0 & 10.6 & 11.5 & 8.7 & 10.4 \\
\hline$T_{1 / 2 \text { calc }}(\mathrm{yr})$ & $1.8 \times 10^{21}$ & $3.3 \times 10^{19}$ & $2.1 \times 10^{18}$ & $1.1 \times 10^{23}$ & $2.3 \times 10^{19}$ & $2.3 \times 10^{19}$ & $5.5 \times 10^{17}$ & $2.2 \times 10^{18}$ \\
\hline
\end{tabular}

${ }^{\mathrm{a}}$ Calculation for spherical nuclei, except ${ }^{150} \mathrm{Nd}$, where deformation $\varepsilon$ is indicated.

${ }^{b}$ Mixing of $\Delta_{33}$ isobar included.

Another special case is ${ }^{48} \mathrm{Ca}$. This doubly magic nucleus is not well suited for the RPA treatment used here. It is known ${ }^{2,11}$ that there is a strong cancellation between the contribution of seniority zero and seniority four states in the final nucleus ${ }^{48} \mathrm{Ti}$. Only a complete shell model calculation can, and indeed does, ${ }^{2}$ account for this effect.

On the other hand, in other cases (with a possible exception of the semi-magic ${ }^{136} \mathrm{Xe}$ ) we expect that the quasiparticle vacuum (seniority zero) is a good approximation of the ground state. Therefore, it is not surprising that we find a reasonable agreement between our results and the shell model calculations of Haxton and Stephenson. ${ }^{2}$

In conclusion, we have studied the effect of pairing, static quadrupole deformation, spin polarization, and the $\Delta_{33}$ isobar admixtures on the double beta decay rates. Our systematic and rather transparent treatment does not remove the discrepancy between the calculated and experimental lifetimes of the $2 v \beta \beta$ decay mode. Without a solution to this problem it will be difficult to interpret the results of searches for the $0 v$ mode in terms of the Majorana neutrino mass and the parameters of the righthanded current weak interactions.

This work was supported by the U.S. Department of Energy under Contract No. DE-AT03-81ER40002.
${ }^{1}$ H. Primakoff and S. P. Rosen, Rep. Prog. Phys. 22, 121 (1959).

2 W. C. Haxton and G. J. Stephenson, Jr., Prog. Part. Nucl. Phys. 12, 409 (1984).

${ }^{3}$ M. Doi, T. Kotani, and E. Takasugi, in Proceedings of the Telemark 84 Conference, edited by V. Barger and D. Cline.

${ }^{4}$ T. Tsuboi, K. Muto, and H. Horie, Phys. Lett. 143B, 293 (1984).

${ }^{5}$ C. Gaarde, Nucl. Phys. A396, 127c (1983).

${ }^{6}$ R. K. Bardin et al., Nucl. Phys. A158, 337 (1970).

${ }^{7}$ F. T. Avignone et al., Phys. Rev. Lett. 50, 721 (1983).

${ }^{8}$ M. K. Moe, A. A. Hahn, and S. R. Elliott, University of California, Irvine, Report UCI-Neutrino No. 133, 1984.

${ }^{9} \mathrm{~T}$. Kirsten, in Science Underground (Los Alamos), Proceedings of the Workshop on Science Underground, AIP Conf. Proc. No. 96, edited by M. N. Nieto et al. (AIP, New York, 1983).

${ }^{10}$ A. A. Klimenko, A. A. Pomansky, and A. A. Smolnikov, in Neutrino '84, edited by K. Kleinknecht and E. A. Paschos
(World Scientific, Singapore, 1984).

${ }^{11}$ L. Zamick and N. Auerbach, Phys. Rev. C 26, 2185 (1982).

${ }^{12}$ W. C. Haxton and G. J. Stephenson, Phys. Rev. C 28, 458 (1983).

${ }^{13}$ A. H. Huffman, Phys. Rev. C 2, 742 (1970).

14J. A. Halbleib and R. A. Sorensen, Nucl. Phys. A98, 542 (1967).

${ }^{15}$ K. Ikeda, S. Fuji, and J. A. Fujita, Phys. Lett. 3, 271 (1963).

${ }^{16}$ K. Grotz, H. V. Klapdor, and J. Metzinger, J. Phys. G 9, L169 (1983); H. V. Klapdor and K. Grotz, Phys. Lett. 142B, 323 (1984).

17J. Krumlinde and P. Moller, Nucl. Phys. A417, 419 (1984).

${ }^{18}$ A. Bohr and B. R. Mottelson, Phys. Lett. 100B, 10 (1981).

${ }^{19}$ K. Grotz, H. V. Klapdor, and J. Metzinger, Phys. Lett. 132B, 22 (1983).

${ }^{20}$ G. F. Bertsch and I. Hamamoto, Phys. Rev. C 26, 1323 (1982). 